Z. klin. Chem. u. klin. Biochem.

7. Jg., S. $162-166$, März 1969

\title{
Untersuchungen über die arteriovenöse Differenz der Alkoholkonzentration im Blut und ihre Beziehung zum Alkoholgehalt des Gehirns
}

\author{
Von J. G. Gostomzyk, B. Dilger und K. Dilger \\ Aus dem Instilut fïr gericbtliche Medizin und Versicherungsmedizin der Universität Freiburg i. Br. \\ (Direletor: Prof. Dr. W. Spann)
}

(Eingegangen am 7. November 1968)

Die arteriovenöse Differenz der Alkoholkonzentration bei intravenöser Alkoholgabe wurde bei 12 Kaninchen mit der Methode der automatischen kontinuierlichen Alkoholbestimmung (Auto-Analyzer, ADH-Verfahren) untersucht. Die narkotisierten Tiere erhielten $0,8 \mathrm{~g}$ pro $\mathrm{kg}$ Alkohol in Form einer 33\%igen Lösung mit einer Infusionsgeschwindigkeit zwischen 5 und 25 Minuten in eine Ohrvene injiziert. Das Blut wurde bei jedem Versuchstier gleichzeitig aus der A. brachialis und V. femoralis abgeleitet und analysiert. Mit Verkürzung der Infusionszeit für den Alkohol wird der Anstieg der Blutalkoholkurve steiler und die Differenz der Alkoholkonzentrationen zwischen Vene und Arterie größer. Im Blut der Arterie wird das Maximum der Alkoholkonzentration mit dem Ende der Infusionszeit erreicht, im Venenblut später. In der Arterie werden während der Anflutungsphase höhere Alkoholkonzentrationen erreicht als in der Vene. Nach dem Diffusionsausgleich sind die Konzentrationen in beiden Gefäßen gleich.

Die Befunde sind geeignet, die in bezug auf die Alkoholkonzentration im Venenblut stärkere Wirkung des Alkohols auf das Zentralnervensystem während der Anflutungsphase zu crklären. Sie werden im Hinblick auf Untersuchungen über die Alkoholkonzentration im Gehirn und:auf EEG-Veränderungen unter Alkohol diskutiert. Die Alkoholwirkung während der Anflutungsphase stimmt besser mit der Blutalkoholkonzentration des arteriellen als mit der des venösen Blutes überein.

\section{Studies on the arterio-venous differences in blood alcobol concentration and its relationship to the alcobol content of brain}

The difference in arterial and venous alcohol concentration during intravenous alcohol infusion was tested on 12 rabbits using the automatic-continuous alcohol analysis (auto-analyser, $\mathrm{ADH}$-system). The anaesthetised rabbits received $0.8 \mathrm{gm}$ alcohol per $\mathrm{kg}$ bodyweight in a $33 \%$ solution, infused in an ear vein over a period of 5 to 25 minutes. Continuous blood analysis from A. brachialis and V. femoralis were made during and after the infusion. A much faster rise in the blood alcohol curve and a greater arterial-venous alcohol concentration difference were found by shorter infusion times. A maximum arterial blood alcohol concentration was reached at the end of the infusion; the maximum in venous blood was reached later. The alcohol concentration during infusion was higher in arterial than venous blood and a higher peak was reached in arterial blood. After diffusion, the alcohol concentrations in arterial and venous blood were the same. These findings explain the greater effect of alcohol, during absorption, on the central nervous system, in relation to the venous blood alcohol concentration. It is interesting to compare, these findings with experiments on alcohol concentration in the brain and resulting EEG changes. The effects of alcohol during the absorption period is more closely related to the arterial than the venous blood alcohol concentration.

Bei gleicher Alkoholkonzentration im Venenblut ist die Alkoholwirkung auf Leistungen des Zentralnervensystems im ansteigenden Teil der Blutalkoholkurve (Anflutungsphase, Resorptionsphase) stärker als im abfallenden Teil (postresorptive Phase) (1). Neben dem psychophysischen Verhalten verändert sich auch die bioelektrische Spontanaktivität des Gehirns (EEG) unter Alkohol (2). Dabei ist die Frequenzerniedrigung und die Amplitudenvergrößerung im Hirnstrombild bei gleicher Alkoholkonzentration im Venenblut während des Anstiegs der Blutalkoholkurve stärker als während ihres Abfalles $(3,4,5,6)$. Als Erklärung für den unterschiedlichen Alkoholeffekt bei gleicher Alkoholkonzentration im Venenblut werden die Steilheit des Anstiegsgradienten ( $\% 0^{-}$-Zuwachs je Zeiteinheit) (3), ein "Überhöhungseffekt" "während der Alkoholanflutung (1) oder eine Adaptation des Gehirns an den Alkoholspiegel (6) diskutiert.

Die vorliegende Arbeit berichtet über den Verlauf von Alkoholkonzentrationskurven im arteriellen und im venösen Blut bei verschieden schneller Alkoholanflutung und über die Bedeutung der Anflutungsgeschwindigkeit für die Alkoholwirkung.

\section{Material und Methoden}

Kaninchen mit einem Körpergewicht von $3072 \pm 314 \mathrm{~g}$ wurden mit 17,8 $\pm 3,2 \mathrm{mg}$ pro $\mathrm{kg}$ Körpergewicht Nembutal i. v. narkotisiert und in Rückenlage fixiert. Sie erhielten $0,8 \mathrm{~g}$ Äthanol pro $\mathrm{kg}$ Körpergewicht in Form einer 33proz. (w/w) wäßr. Lösung mit Hilfe einer Druckpumpe kontinuierlich in eine Ohrvene injiziert, wobei die Einlaufzeit zwischen 5 und 25 Min. variiert wurde.

Die Bestimmung der Blutalkoholkonzentration (BAK) erfolgte nach dem $A D H^{1}$ )-Verfahren mit Hilfe der kontinuierlichen automatischen Bestimmung nach Lerthoff (7). Dieses Verfahren ermöglicht es, den kontinuierlich mittels einer Saugpumpe aus einem Gefäß abgeleiteten Blutstrom fortlaufend zu analysieren, so daß alle Veränderungen der Alkoholkonzentration im entsprechenden Gefäß exfaßt werden.

Die Methode wurde dahingehend modifiziert, daß wir 2 AnalysenWege parallel laufen ließen. Für jedes System wurde eine Eichkurve mit standardisierten Alkohollösungen geschrieben. Um eine Blutgerinnung im Entnahme- und Analyse-System zu vermeiden, ethielten die Tiere 1717 干538 E. Heparin pro $90 \mathrm{Min}$. in die Ohrvene injiziert. Pro Stunde wurden insgesamt etwạ $40 \mathrm{~m} /$ Blut abgesaugt.

Das arterielle Blut wurde mittels eines in die A. brachialis eingebundenen Katheters entnommen, wobei die Offnung des Katheters

1) $\mathrm{ADH}=$ Alkohol-Dehydrogenase $=$ Alkohol: NAD Oxydoreduktase (EC 1.1.1.1). 
zum Herzen hin gerichtet war. Das venöse Blut wurde aus der V. femoralis, die an der Innenseite des Oberschenkels der Tiere freigelegt wurde, durch einen Katheter entnommen, wobei die Katheteröffnung zur Peripherie zeigte.

\section{Ergebnisse}

Die Abbildungen 1-4 zeigen die Mittelwertskurven der Blutalkoholkonzentrationen in der A. brachialis und in der V. femoralis bei Variation der Infusionszeiten für die Alkohollösung zwischen 5 und 25 Min. Eingezeichnet ist weiterhin die einfache Streuung der Alkoholkonzentrationen, die für verschiedene Zeitpunkte berechnet wurde.

Der Anstieg der Blutalkoholkonzentration $\left(\% / 00^{-Z u-~}\right.$ wachs je Zeiteinheit) verläuft sowohl in der Arterie als

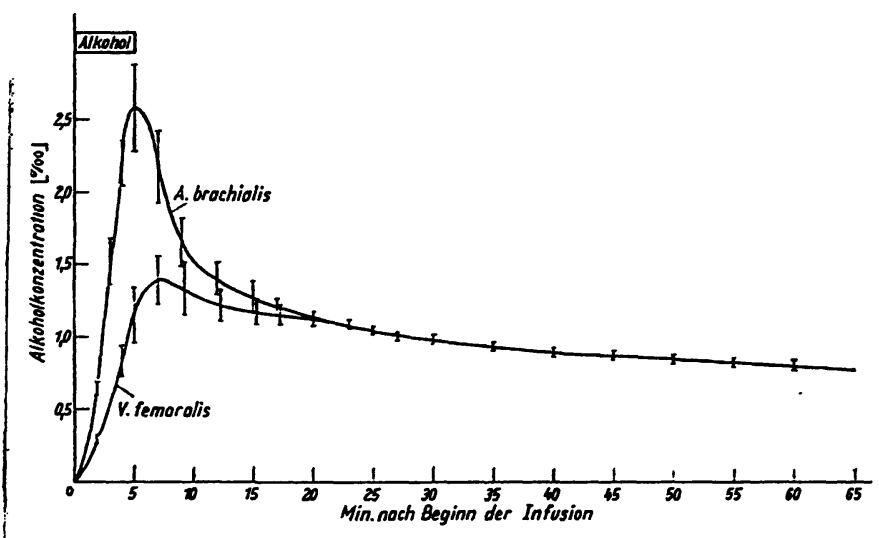

Abb. 1

Verhalten der Blutalkoholkonzentrationen in der A. brachialis und in der $V$. femoralis bei kontinuierlicher Infusion einer 33proz. ( $w / w)$ Alkohollösung $(0,8 \mathrm{~g} / \mathrm{kg}$ Körpergewicht) innerhalb von $5 \mathrm{Min}$. in eine Ohrvene beim Kaninchen. Mittelwertskurve aus 3 Versuchen

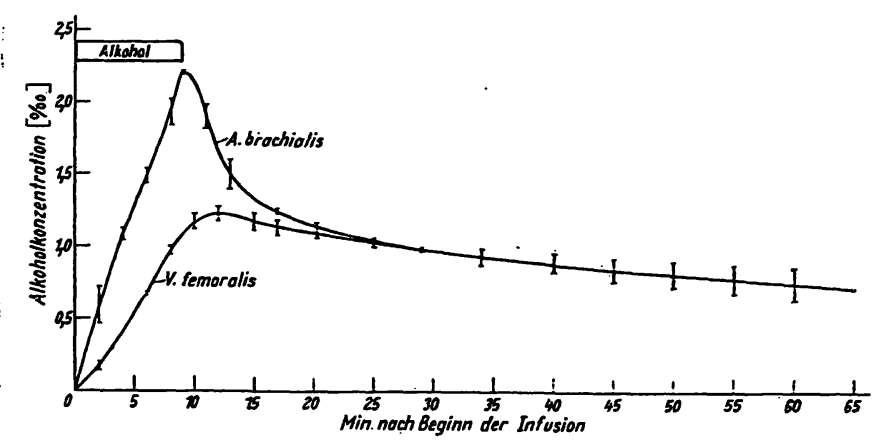

Abb. 2 Verhalten der Blutalkoholkonzentrationen in der A. brachialis und
in der $\mathrm{V}$. femoralis bei kontinuierlicher Infusion einer 33 proz. ( $w / w)$ in der $\mathrm{V}$. femoralis bei kontinuierlicher Infusion einer 33 proz. (W/W
Alkohollösung $\cdot(0,8 \mathrm{~g} / \mathrm{kg}$ Körpergewicht) innerhalb von $9 \mathrm{Min}$. in eine

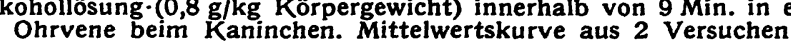

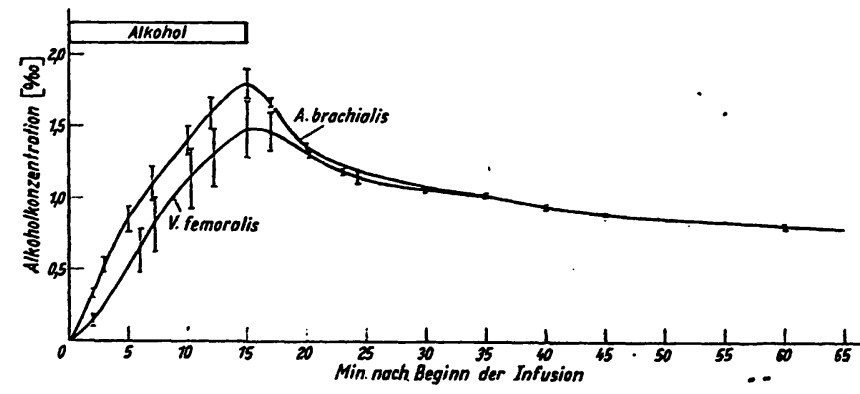

Abb. 3

Verhalten der Blutalkoholkonzentrationen in der $A$. brachialis und in der $\mathrm{V}$. femoralis bei kontinuierlicher Infusion einer 33proz. (w/w) eine Ohrvene beim Kaninchen. Mittelwertskurve aus 2 versuchen

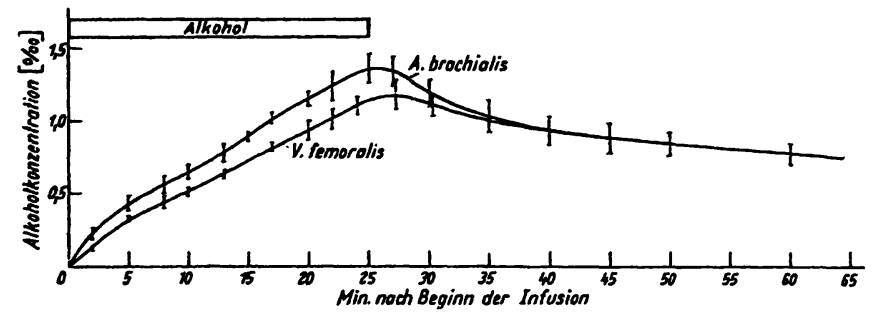

Abb. 4

Verhalten der Blutalkoholkonzentrationen in der $A$. brachialis und in der $\mathrm{V}$. femoralis bei kontinuierlicher Infusion einer 33proz. (w/w) Alkohollösung $(0,8 \mathrm{~g} / \mathrm{kg}$ Körpergewicht) innerhalb von $25 \mathrm{Min}$. in eine Ohrvene beim Kaninchen. Mittelwertskurve aus 2 Versuchen

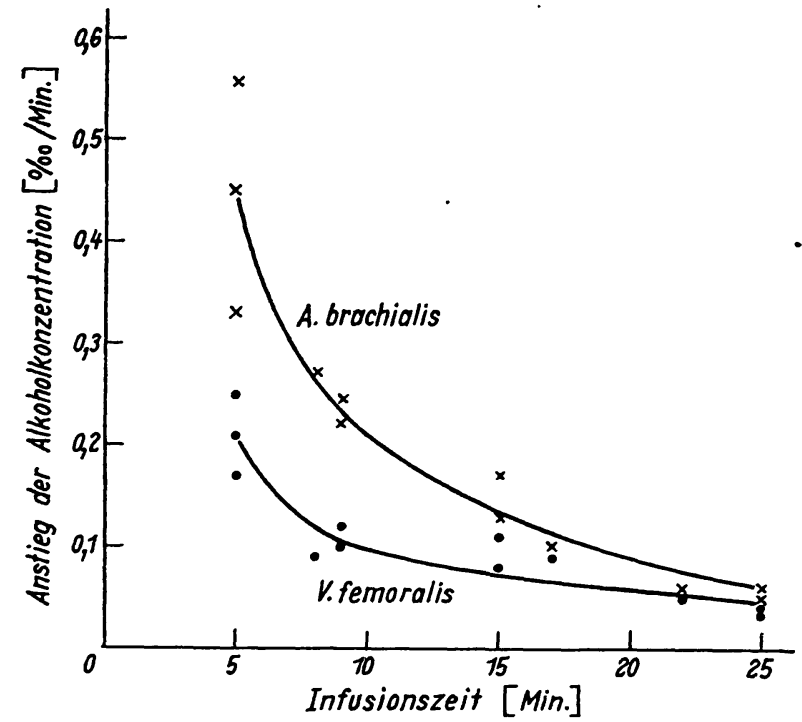

Abb. 5

Verhalten des Anstiegs der Blutalkoholkonzentration (Promillezuwachs/Minute) in Abhângigkeit von der Anflutungsgeschwindigkeit (Infusionszeit) des Alkohols. Auswertung von 12 Versuchen mit Variation der Infusionszeit zwischen 5 und 25 Min. Verabreicht wurden jeweils $0,8 \mathrm{~g} / \mathrm{kg}$ Körpergewicht Äthanol in Form einer 33proz. $(w / w)$ wäßr. Lösung

auch in der Vene mit zunehmender Einlaufzeit erwartungsgemä $ß$ flacher. Abbildung 5 zeigt die Abhängigkeit der $\% 0^{-Z u w a c h s r a t e ~ v o n ~ d e r ~ I n f u s i o n s z e i t . ~ D a s ~}$ Maximum der arteriellen Blutalkoholkonzentration wird mit Ende der Infusionszeit erreicht. Der Höhepunkt der Blutalkoholkonzentration in der Vene liegt zeitlich etwas hinter dem Maximum in der Arterie.

Tabelle 1 gibt die erreichten maximalen Alkoholkonzentrationen $\left(C_{\max }\right)$ im Blut der A. brachialis und in $\operatorname{der} \mathrm{V}$. femoralis bei verschieden langer Einlaufzeit wieder. Weiterhin wurde die hypothetische Blutalkoholkonzentration für den Zeitpunkt des Beginns der Alkoholinfusion $\left(\mathrm{C}_{0}\right.$-Wert) berechnet. Bei dieser Berechnung wurde jeweils der individuelle Abbauwert, der aus dem Abfall der Blutalkoholkurve zwischen 40 und $60 \mathrm{Min}$. abgeleitet worden war, verwendet. Die Differenz $\mathrm{C}_{\max }-\mathrm{C}_{0}$ ist Ausdruck des mit Ende der Alkoholinfusion noch nicht erreichten Diffusionsausgleiches der Alkoholkonzentrationen. Die Differenz $C_{\max }-C_{0}$ ist im arteriellen Blut größer als im Blut der peripheren Vene und zeigt für die arterielle Blutalkoholkonzentration eine deutliche Abhängigkeit von der Einlaufgeschwindigkeit der Alkohollösung. Für die Wirkung des Alkohols auf bestimmte Organe während der An- 
Tab. 1

In der ersten Spalte sind die hypothetischen Blutalkoholkonzentrationen $\left(C_{0}\right)$ für den Zeitpunkt des Beginns der Alkoholinfusion angegeben. Sie wurdenl durch Rückrechnung aus dem Abfall der Blutalkoholkonzentrationskurve zwischen 40 bis 60 Min. nach Beginn der Infusionszeit ermittelt. $C_{0}$ ist in arteriellen und venösen Blut gleich. Die Differenzen zwischen maximaler Blutalhoholkonzentration ( $C_{\max }$ ) in der $A$. brachialis und dem $C_{0}$-Wert sowie zwischen den maximalen Blutalkoholkonzentrationen in $\operatorname{der} A$. brachialis und der $V$. femoralis ( $A$. $C_{\max }-V$. $\left.C_{m a x}\right)$ werden bei kürzeren Infusionszeiten einer gleichen Alkoholdosis $(0,8 \mathrm{~g} / \mathrm{kg}$ Körpergewicht, 33proz. (w/w) Lösung) größer. Die Blutalkoholkonzentrationen nach Ausgleich der arteriovenösen Differenz, z. B. 45 bzw. 60 Min. nach Beginn der Alkoholinfusion, sind.unabhängig von den $C_{\text {max }}-$ Werten. Die Untersuchungen wurden an 12 Kaninchen durchgeführt

\begin{tabular}{|c|c|c|c|c|c|c|c|c|}
\hline \multirow[b]{2}{*}{$\begin{array}{l}\text { Dauer der } \\
\text { Alkoholinfusion }\end{array}$} & \multirow[b]{2}{*}{ A. $=\mathrm{V}$} & \multicolumn{5}{|c|}{ Alkoholkonzentration:in \% } & \multirow{2}{*}{\multicolumn{2}{|c|}{$\begin{array}{l}\text { Zeit nach Beginn der Infusion } \\
\times \quad 45 \text { Min. } 60 \text { Min. }\end{array}$}} \\
\hline & & $\mathrm{C}_{\max }$ A. bre & $\stackrel{\mathrm{C}_{\max }}{\text { achialis }}-\mathrm{C}_{0}$ & $c_{\max }$ V. fer & $\stackrel{\text { noralis }}{\mathrm{C}_{\max }}-\mathrm{C}_{0}$ & $\mathrm{AC}_{\max }-\mathrm{VC}_{\max }$ & & \\
\hline $\begin{array}{l}5 \text { Min. } \\
5 \text { Min. } \\
5 \text { Min. }\end{array}$ & $\begin{array}{l}1,10 \\
1,03 \\
1,06\end{array}$ & $\begin{array}{l}2,25 \\
2,67 \\
2,82\end{array}$ & $\begin{array}{l}1,15 \\
1,64 \\
1,76\end{array}$ & $\begin{array}{l}1,20 \\
1,50 \\
1,48\end{array}$ & $\begin{array}{l}0,10 \\
0,47 \\
0,42\end{array}$ & $\begin{array}{l}1,05 \\
1,17 \\
1,34\end{array}$ & $\begin{array}{l}0,90 \\
0,84 \\
0,88\end{array}$ & $\begin{array}{l}0,82 \\
0,76 \\
0,82\end{array}$ \\
\hline $\bar{x} \pm s$ & $1,06 \pm 0,04$ & $2,58 \pm 0,30$ & $1,52 \pm 0,30$ & $1,39 \pm 0,17$ & $0,33 \pm 0,20$ & $1,18 \pm 0,14$ & $0,87 \pm 0,03$ & $0,80 \pm 0,03$ \\
\hline $\begin{array}{l}9 \mathrm{Min} . \\
9 \mathrm{Min} . \\
8 \mathrm{Min} .\end{array}$ & $\begin{array}{l}1,04 \\
1,07 \\
1,24\end{array}$ & $\begin{array}{l}2,22 \\
2,20 \\
2,19\end{array}$ & $\begin{array}{l}1,18 \\
1,13 \\
0,95\end{array}$ & $\begin{array}{l}1,17 \\
1,29 \\
1,13\end{array}$ & $\begin{array}{r}0,13 \\
0,21 \\
-0,11\end{array}$ & $\begin{array}{l}1,05 \\
0,92 \\
1,06\end{array}$ & $\begin{array}{l}0,92 \\
0,76 \\
0,98\end{array}$ & $\begin{array}{l}0,86 \\
0,64 \\
0,88\end{array}$ \\
\hline $\bar{x} \pm s$ & $1,12 \pm 0,11$ & $2,20 \pm 0,02$ & $1,09 \pm 0,10$ & $1,19 \pm 0,03$ & $0,15 \pm 0,05$ & $1,01 \pm 0,08$ & $0,89 \pm 0,11$ & $0,79 \pm 0,13$ \\
\hline $\begin{array}{l}15 \text { Min. } \\
15 \text { Min. } \\
17 \text { Min. }\end{array}$ & $\begin{array}{l}1,12 \\
1,11 \\
1,23\end{array}$ & $\begin{array}{l}1,72 \\
1,90 \\
1,66\end{array}$ & $\begin{array}{l}0,60 \\
0,79 \\
0,43\end{array}$ & $\begin{array}{l}1,36 \\
1,65 \\
1,48\end{array}$ & $\begin{array}{l}0,24 \\
0,54 \\
0,25\end{array}$ & $\begin{array}{l}0,36 \\
0,25 \\
0,18\end{array}$ & $\begin{array}{r}0,90 \\
0,88 \\
0,98\end{array}$ & $\begin{array}{l}0,82 \\
0,80 \\
0,89\end{array}$ \\
\hline $\bar{x} \pm s$ & $1,15 \pm 0,07$ & $1,76 \pm 0,13$ & $0,61 \pm 0,18$ & $1,49 \pm 0,15$ & $0,34 \pm 0,17$ & $0,26 \pm 0,08$ & $0,92 \pm 0,05$ & $0,84 \pm 0,05$ \\
\hline $\begin{array}{l}25 \text { Min. } \\
25 \text { Min. } \\
22 \text { Min. }\end{array}$ & $\begin{array}{l}1,33 \\
1,09 \\
1,18\end{array}$ & $\begin{array}{l}1,56 \\
1,37 \\
1,55\end{array}$ & $\begin{array}{l}0,23 \\
0,28 \\
0,37\end{array}$ & $\begin{array}{l}1,28 \\
1,08 \\
1,28\end{array}$ & $\begin{array}{r}-0,05 \\
-0,01 \\
0,10\end{array}$ & $\begin{array}{l}0,28 \\
0,29 \\
0,27\end{array}$ & $\begin{array}{l}0,98 \\
0,79 \\
0,96\end{array}$ & $\begin{array}{l}0,86 \\
0,68 \\
0,88\end{array}$ \\
\hline $\bar{x} \pm s$ & $1,20 \pm 0,12$ & $1,49 \pm 0,11$ & $0,29 \pm 0,07$ & $1,21 \pm 0,12$ & $0,05 \pm 0,05$ & $0,28 \pm 0,01$ & $0,91 \pm 0,06$ & $0,81 \pm 0,11$ \\
\hline
\end{tabular}

flutungsphase dürfte die Differenz zwischen $C_{\max }$ in der Arterie $-C_{\max }$ in der Vene, die bei kürzerer Anflutungszeit größer wird, besondere Bedeutung haben. Nach dem Ausgleich der arteriovenösen Differenz der Alkoholkonzentrationen ist die Höhe der Blutalkoholkonzentration bei der von uns gegebenen Alkoholmenge praktisch von der Einlaufzeit des Alkohols unabhängig. Bei den 3 Versuchstieren mit einer Infusionszeit von $5 \mathrm{Min}$. betrug die Blutalkoholkonzentration 45 Min. nach Beginn der Infusion $0,87 \pm 0,03 \%$ und $60 \mathrm{Min}$. danach $0,80 \pm 0,03 \%$. Bei einer Infusionszeit von 22 bzw. 25 Min. betrug die Blutalkoholkonzentration bei 3 Tieren $45 \mathrm{Min}$. nach Beginn der Einlaufzeit $0,91 \pm 0,06 \%$ und 60 Min. nach dem Beginn $0,80 \pm$ $0,11 \%$. Nach dem t-Test kann die Hypothese, daß die Mittelwerte beider Gruppen (jeweils $\mathrm{n}=3$ ) der gleichen Grundgesamtheit angehören, für die 45 Minuten-Werte mit einem $t=1,03$ und für die 60 Minuten-Werte mit einem $t=0,153$ nicht verworfen werden.

\section{Diskussion}

Die Untersuchungsergebnisse über den Verlauf der Blutalkoholkonzentrationskurve in $\operatorname{der} A$. brachialis und in der $\mathrm{V}$. femoralis zeigen, daß die im Blut der Venen einer Extremität festgestellte Alkoholkonzentration während der Anflutungsphase des Alkohols nicht für die Alkoholkonzentration im Blut anderer Abschnitte des Gefäßsystems repräsentativ ist, Sie bestätigen Untersuchungen von Haggard und Greenberg (8), PAINe und Mitarbeiter (9), sowie Forney und Mitarbeiter (10), die in Einzelblutproben aus verschiedenen Gebieten des Gefäßsystems während des ansteigenden Teiles der Blutalkoholkurve unterschiedliche Alkoholkonzentrationen fanden. Dabei waren die Alkoholkonzentrationen im rechten Herzen, in der A. pulmonalis und in der V. jugularis bis zum Erreichen des Konzentrationsmaximums etwa gleich hoch, etwas niedriger war dagegen die Alkoholkonzentration im Kapillarblut der Haut und deutlich niedriger im Blut der V. femoralis.

Die von uns angewendete Methode mit gleichzeitiger kontinuierlicher Entnahme und Analyse von Blut aus einer Arterie und einer Vene ergab, daß die arterielle Blutalkoholkonzentration höhere Maximalwerte erreicht als die venöse. Weiterhin liegt der Gipfel der Blutalkoholkonzentration in der Arterie zeitlich vor dem Maximum im Blut der peripheren Vene. Die maximale Alkoholkonzentration im arteriellen Blut wird mit Ende der Infusionszeit des Alkohols erreicht.

Die unterschiedliche Alkoholkonzentration im venösen Stromgebiet dürfte durch den verschieden schnellen Ausgleich zwischen arterieller Blutalkoholkonzentration und der Alkoholkonzentration von bestimmten Geweben, je nach dem Grad ihrer Durchblutung, bedingt sein. In Geweben mit hohem Durchströmungsvolumen, wie z. B. im Gehirn und in den Nieren, wird der Konzentrationsausgleich sehr bald erreicht. Der periphere Muskel hat dagegen ein geringeres Durchblutungsvolumen. Entsprechend wird beim Konzentrationsausgleich aus dem Blut mehr Alkohol in das Muskelgewebe diffundieren, da die Muskelmasse für. die im Blutstrom zugeführte Alkoholmenge ein verhältnismäßig großes Lösungsvolumen darstellt. Daraus resultiert, daß die Alkoholkonzentration im Muskelgewebe verhältnismäßig langsam ansteigt und die Alkoholkonzentration im dazugehörigen Venenblut entsprechend niedrig liegt.

Unter Verwendung von Äthanol-[1-14C] konnte in Versuchen an Mäusen gezeigt werden, daß die Alkoholkonzentrationen im Nieren-, Hirn- und Lungengewebe während der Anflutungsphase etwa gleich sind. Diese Alkoholkonzentrationen lassen sich unter Berücksichti- 
gung der Kreislaufverhältnisse erklären. Das aus dem Bauchraum kommende alkoholreiche Blut durchströmt zuerst die Lunge. Das arterialisierte und alkoholreiche Blut führt dann im Gehirn und in den Nieren zu einem schnellen Anstieg der Alkoholkonzentration, da diese Organe cinen hohen Anteil am Herz-Minutenvolumen und ein großes Durchblutungsvolumen haben. Dagegen liegt die Alkoholkonzentration im M. quadriceps während der Anflutungsphase statistisch signifikant niedriger. $\mathrm{Da}$ Alkohol im Organismus frei diffundiert und nicht gegen ein Konzentrationsgefälle angereichert wird, muß die Alkoholkonzentration im Lungengewebe der Alkoholkonzentration im arteriellen Blut entsprechen. Von der Alkoholkonzentration im Muskelgewebe wurde auf die Alkoholkonzentration im dazugehörigen Venenblut geschlossen. Aus den Alkoholkon-

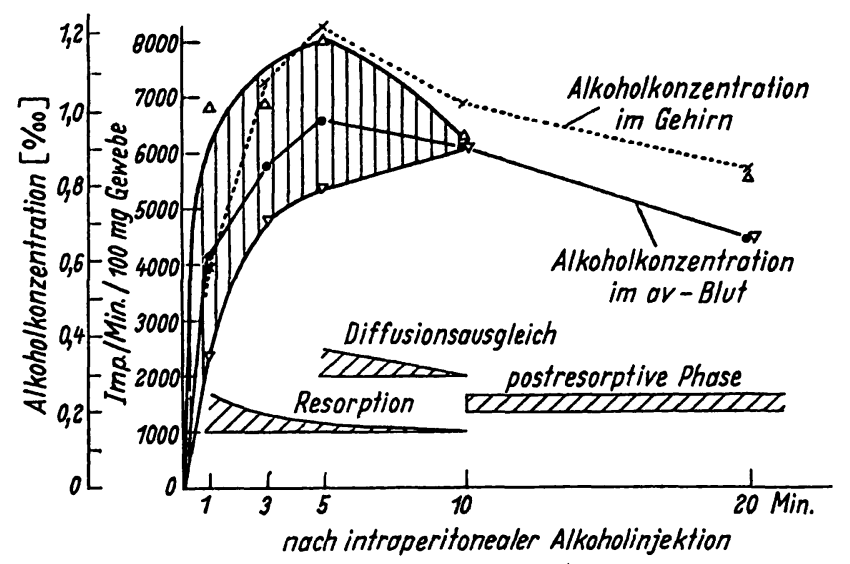

Abb. 6

Die Abbildung zeigt die Ableitung der arteriovenösen Differenz der Blutalkoholkonzentrationen aus der Alkoholkonzentration im Lungengewebe $\hat{\widehat{a}}$ (슬 Arterie) und aus der im Gewebe des $M$. quadriceps $\nabla$ 슬. Weiter sind die Alkoholkonzentrationen für das Hirngewebe und für das nach Dekapitation der Mäuse aus den Halsgefäßen abfließenden arteriovenösen Mischblut angegeben. Die angegebenen Werte sind Mittelwerte aus Versuchen an jeweils 5 Mäusen. Die $25 \mathrm{~g}$

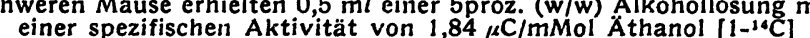

zentrationen in den Organen wurde der Bereich der arteriovenösen Differenz der Alkoholkonzentrationen abgeleitet (Abb. 6). Das Hirngewebe zeigte in diesen Versuchen eine Alkoholkonzentration, die sehr bald der Alkoholkonzentration im Lungengewebe und damit der des arteriellen Blutes entsprach (11).

Die aus dem Alkoholgehalt des Lungengewebes für das arterielle Blut und aus dem Alkoholgehalt des $M$. quadriceps für das Blut der peripheren Vene abgeleiteten Konzentrationskurven zeigen einen Verlauf, der mit den kontinuierlich bestimmten Alkoholkonzentrationskurven in $\operatorname{der} \mathrm{A}$. brachialis und $\operatorname{der} \mathrm{V}$. femoralis, insbesondere bei kurzer Infusionszeit des Alkohols (Abb. 1 und 2), vergleichbar erscheint. So erreicht die absolute Alkoholkonzentration im. Lungen-, Nieren- und Hirngewebe höhere Werte als die Alkoholkonzentration im Muskelgewebe. Die hohen Alkoholkonzentrationen im Gewebe entsprechen der Konzentration des arteriellen Blutes. Das Maximum der Alkoholkonzentration im Muskelgewebe wird erst erreicht, wenn die Konzentration im Lungen-, Nieren- und Hirngewebe nach Abschluß der Resorption im Zuge des Diffusionsausgleiches der
Konzentrationen zwischen den verschiedenen Organen bereits wieder abfällt. Dem Verlauf der Alkoholkonzentrationskurve im M. quadriceps ist der Verlauf der Konzentrationskurve im Blut der V. femoralis sehr ähnlich.

Nach Erreichen des Diffusionsausgleiches ist der Alkoholgehalt in den Organen lediglich von ihrem Wassergchalt abhängig, da der stark hydrophile Äthylalkohol praktisch nur im Gewebewasser verteilt ist $(12,13)$. Dementsprechend wat die Blutalkoholkonzentration $45 \mathrm{Min}$ bzw. $60 \mathrm{Min}$ nach Versuchsbeginn bei allen Versuchstieren praktisch gleich, unabhängig von den sehr unterschiedlichen Alkoholkonzentrationen im arteriellen Blut, die in der Anflutungsphase in Abhängigkeit von verschieden schnellen Einlaufgeschwindigkeiten mit gleich großen Alkoholdosen crreicht worden waren. In der postresorptiven Phase ist die Höhe der Blutalkoholkonzentration von der verabreichten Alkoholmenge, jedoch nicht von der Anflutungsgeschwindigkeit abhängig.

Kakolewski und Himwich (6) haben in einer Versuchsanordnung, deren zeitlicher Ablauf der unsrigen sehr ähnlich war, das EEG von Kaninchen abgeleitet. Sie gaben $3000 \mathrm{~g}$ schweren Kaninchen $0,8 \mathrm{~g}$ Alkohol pro kg Körpergewicht in Form einer 20proz. Lösung i. v. innerhalb von 5-8 Min. In den ersten $15 \mathrm{Min}$. nach der Injektion sahen sie zunehmende Veränderungen im EEG, die sich in den folgenden $45 \mathrm{Min}$. allmählich zurückbildeten.

Quantitative Beziehungen zwischen Blutalkoholkonzentration und Veränderungen im Hirnstrombild untersuchten CASPERS und ABELE (3) an 20 Versuchspersonen. Während einer Trinkzeit von $30 \mathrm{Min}$. erhielten die Versuchspersonen 0,4-1,9 g Alkohol pro Kg Körpergewicht in Form eines 38proz. Doppelkorns. Zur Blutalkoholbestimmung benutzten sie Kapillarblut aus der Fingerbeere. Die beobachteten Rhythmusveränderungen im EEG waren bei einer Blutalkoholkonzentration quantitativ identisch, die im ansteigenden Teil der Blutalkoholkurve um $0,3-0,4 \%$ niedriger lag als im abfallenden Teil. Besonders auffallend war der Zeitpunkt der Wirkungsmaxima, die in 50\% der Fälle 5-10 Min. vor dem Gipfelpunkt der Blutalkoholkurve lagen, in weiteren $45 \%$ praktisch mit dem Maximum der Blutalkoholkurve zusammenfielen. Die Variation der Trinkzeiten auf $10 \mathrm{bzw}$. $60 \mathrm{Min}$. ergab, daß der Grad der Änderung des Hirnstrombildes von der Höhe des erreichten $\% 0^{-W e r t e s}$ und von der Steilheit des Konzentrationsanstiegs abhängt.

Der Verlauf der beschriebenen EEG-Veränderungen nach Alkoholgabe zeigt eine auffallende Übereinstimmung mit dem Ablauf der Blutalkoholkonzentration im arteriellen Blut in unseren Kurven. Durch eine gleiche Alkoholmenge werden bei kurzer Anflutungszeit mit stejilem Anstieg der Blutalkoholkurve kurzzeitig wesentlich höhere Alkoholkonzentrationen im arteriellen Blut erzielt als bei flachem Anstieg, wobei das Maximum der Alkoholkonzentration in der Arterie zeitlich vor dem Gipfel der Blutalkoholkonzentration im peripheren Venenblut liegt. 
In unseren Versuchen lag bei intravenöser Infusion von Alkohol mit Variation der Einlaufzeit zwischen 5 bis $25 \mathrm{Min}$. die Alkoholkonzentration im arteriellen Blut während der Anflutungsphase in jedem Falle über der Alkoholkonzentration im Blut der V. femoralis. Zur forensischen Beurteilung der Trunkenheit wird vornehmlich das Blut aus einer Extremitätenvene verwendet.
Die in der Anflutungsphase in bezug auf die Alkoholkonzentration des peripheren Venenblutes zu beobachtende stärkere psychophysische Wirkung des Alkohols läßt sich gut aus der arteriovenösen Differenz der Alkoholkonzentrationen und dem schriellen Ausgleich zwischen arterieller Alkoholkonzentration und Alkoholkonzentration im Hirngewebe erklären.

\section{Literatur}

1. BsCHOR, F., Dtsch. Zschr. gerichtl. Med. 40, 399 (1951). 2. Davis, P. A., F. A. Gibbs, H. Davis, H. W. Jetrer und L. S. Towndridge, Quart. J. Alcohol 1, 621 (1941). - 3. Caspers, H. und G. ABELE, Dtsch. Zschr. getichtl. Med. 45, 492 (1956). 4. Von Hedenström, J. und O. Schmidr, Dtsch. Zschr. getichtl. Med. 40, 234 (1951). - 5. Müller, E. und J. Rutenfranz, Dtsch. Zschr. gerichtl. Med. 50, 54 (1960). - 6. KAKOLEwsKI, J. W. und H. E. Hiмwich, Quart. J. Alcohol 29, 290 (1968). -
7. LeIthoff, H., Blutalkohol 2, 541 (1964). - 8. HAGGARD, H. W. und L. H. Greenderg, J. Pharmacol. 52, 150 (1934). - 9. Payne, J. P., W. D. Hille und N. W. KING, Brit. med. J. 196 (1966). 10. Forney, R. B., F. W. Hughes, R. N. Harger und A. Richards Quart. J. Alcohol 25, 205 (1964). - 11. GostomzYK; J. G. und C. Strefrer, Blutalkohol (im Druck). - 12. GrüNER, O., Dtsch. Zschr. gerichtl. Med. 46, 53 (1957). - 13. GRÜNER, O., Dtsch. Zschr. gerichtl. Med. 49, 235 (1959).
Dr. J. G. Gostomzyk 78 Freiburg i. Br. Albertstr. 9 